\title{
Antidiabetic Effects of the Different Fractions of Ethanolic Extracts of Ocimum sanctum in Normal and Alloxan Induced Diabetic Rats
}

\author{
M. R. I. Khan ${ }^{1}$, M. A. Islam ${ }^{1}$, M. S. Hossain ${ }^{2}$, M. Asadujjaman' ${ }^{2}$, M. I. I. Wahed ${ }^{3}$, B. M. \\ Rahman $^{3}$, A. S. M Anisuzzaman ${ }^{3}$, S. M. Shaheen ${ }^{3}$ and Maruf Ahmed ${ }^{3 *}$ \\ ${ }^{1}$ Department of Pharmacy, International Islamic University Chittagong, Chittagong-4203 \\ ${ }^{2}$ Department of Pharmacy, Atish Dipankar University of Science and Technology, Dhaka-1212 \\ ${ }^{3}$ Department of Pharmacy, University of Rajshahi, Rajshahi-6205, Bangladesh
}

Received 2 July 2009, accepted in revised form 16 November 2009

\begin{abstract}
The antidiabetic effects of Ethyl acetate (Et-Ac), Petroleum-ether (Pet-ether), and Chloroform fractions from ethanolic extract of the leaves of Ocimum sanctum were investigated in normal and alloxan induced diabetic rats (AIDRs). The effect of these fractions $(200 \mathrm{mg} / \mathrm{kg}$ body weight i.p) on fasting blood glucose (FBG), total cholesterol (TC), triglyceride (TG), serum glutamate oxaloacetate transaminases, serum glutamate pyruvate transaminases (SGOT, SGPT) level, and liver glycogen content were investigated in AIDRs and found significant effects. The most significant reduction of FBG level of around $80.19 \%$ was observed for Et-Ac fraction in AIDRs. A significant reduction $(p<0.01)$ in serum TC and TG level of $54.49 \%$ and $79.78 \%$ respectively was also found for Et-Ac fraction of $O$. sanctum. The hypoglycemic and hypolipidemic activities were comparable to metformin $\mathrm{HCl}(150 \mathrm{mg} / \mathrm{kg})$. In severely diabetic rats, liver glycogen content was decreased by $50.60 \%$. Administration of these fractions to the AIDRs resulted in the significant elevation of liver glycogen content. In diabetic rats, SGOT and SGPT levels were significantly elevated that were further reduced after intraperitoneal administration of these fractions. These results indicate that different fractions of $O$. sanctum have favorable effects in bringing down the severity of diabetes together with hepatoprotectivity.
\end{abstract}

Keywords: Fasting blood glucose; Hypolipidemic; Serum glutamate oxaloacetate transaminases; Serum glutamate pyruvate transaminases; Hepatoprotectivity.

(C) 2010 JSR Publications. ISSN: 2070-0237 (Print); 2070-0245 (Online). All rights reserved.

DOI: 10.3329 /jsr.v2i1.2769 J. Sci. Res. 2 (1), 158-168 (2010)

\section{Introduction}

Diabetes is a major threat to global public health that is rapidly getting worse and biggest impact in on adult of working age in developing countries [1]. There are an estimated 246 million people with diabetes in the world, of whom about $80 \%$ reside in developing countries [2]. Although diabetes is often not recorded as the cause of death, globally, it is believed to be the fifth leading cause of death in 2000 after communicable diseases,

\footnotetext{
* Corresponding author: marufahmed@ru.ac.bd
} 
cardiovascular disease, cancer and injuries [3]. The number of cases of non -insulin dependent diabetes mellitus (Type-2) has increased dramatically due to the changes in lifestyle, increasing prevalence of obesity, and ageing of populations. The most disturbing trend is a shift in age of onset of diabetes to a younger age in recent years. This presents a serious challenge to the healthcare system because, at the peak of their working career, people with diabetes have an excess risk of mortality and morbidity compared with those without diabetes [4-6]. Indeed, over two-thirds of deaths attributable to diabetes occur in developing countries [7].

According to the World Health Organization, more than $70 \%$ of the world's population must use traditional medicine to satisfy their principal health needs. A great number of medicinal plants used in the control of the diabetes mellitus have been reported [8]. There are various medicinal plants in the world, which are the potential sources of the drugs. The discovery of the widely used hypoglycemic drug, metformin (N, Ndimethylguanylguanidine) came from the traditional approach through the use of Galega officinialis [9].

Traditionally various plants are being used to treat diabetic patients. It is believed that, herbal medicine has little side effects as well as it requires no cost in few cases. Thus it can solve the economic problem of the poor. Now a day, the scientists and researchers are looking for natural plant products by research all over the world and a large number of the evidence have shown the immense potential of medicinal plants used traditionally. Bangladesh is full of medicinal plants, which are used by the people for the treatment of various diseases even at this modern era. In Bangladesh about 5 million people are affected with diabetes for various reasons. In recent years the popularity of complementary medicines has increased. Dietary measures and traditional plant therapies as prescribed by Ayurvedic and other indigenous systems of medicine have been used commonly in India.

Ocimum sanctum Linn. (Labiate), commonly known as holy basil, is an herbaceous plant found throughout the south Asian region is used medicinally in catarrhal bronchitis, bronchial asthma, dysentery, dyspepsia, skin diseases, chronic fever, haemorrhage and helminthiasis, and topically for ringworms. [10-13]. The leaf is claimed to possess hypoglycemic and antihyperglycaemic effects in experimental animals [14]. The objective of the present work was to make an analysis of the ethno botanical information on the medicinal plants used in diabetes mellitus control, and of the results obtained in the investigation of the hypoglycemic Activity of such plants. In the present study the antidiabetic properties of the leaves of $O$. sanctum was assessed by evaluating the comparative antihyperglycemic and hypolipidemic activities of this in normal, glucose induced and alloxan induced diabetic rats.

\section{Materials and Methods}

\subsection{Plant materials}

The fresh leaves of $O$. sanctum (locally named 'Tulsi') were collected from medicinal plant garden, Department of Pharmacy, University of Rajshahi, Rajshahi. After dried 
completely under the mild sun and grinded to a coarse powder they were used for cold extraction. The authenticity of the $O$. sanctum was identified by Mr. AHM Mahbubur Rahman, Department of Botany, University of Rajshahi. A voucher specimen, collection \# 60 , dated 8/25/2007 has been kept in the Department of Botany, University of Rajshahi, Rajshahi.

\subsection{Preparation and fractionation of crude extracts}

The coarse powder was submerged in ethyl alcohol (96\%) and allowed to stand for several days (7-10) with occasional shaking and stirring. When the solvent become concentrated, the liquid alcohol content was filtered through cotton and then through filter paper (Whatman filter paper \#1). Then the solvents were allowed to evaporate using rotary evaporator at temperature $40-45^{\circ} \mathrm{C}$. Thus the highly concentrated crude extracts were obtained. They were then fractionated using Et-Ac, Pet-ether, and $\mathrm{CHCl}_{3}$. The dried fractionated extracts were then preserved in the refrigerator for the experimental use.

\subsection{Drugs and chemicals}

The active drug, Metformin hydrochloride was the generous gift samples from Square Pharmaceuticals Ltd., Pabna Bangladesh. Total cholesterol (TC) and triglyceride (TG) wet reagent diagnostic kits were the products of Cresent diagnostic kits. Alloxan was purchased from Sisco Research Laboratories Pvt. Ltd. Mumbai, India. SGPT and SGOT wet reagent diagnostic kits were purchased from AMP Medizintechnik GmbH; Austria.

\subsection{Experimental design}

In the experiment, a total of 55 long-Evans rats about 120-150 gm; age 6-8 weeks (25 diabetic surviving rats, 30 normal rats) were used. Group II to Group VI was prepared for testing antihyperglycemic effects after chemical diabetes. Group I received only vehicle (DMSO). Group II was selected for diabetic control, which does not receive either metformin, or plant extracts. Group III stands for metformin control group in which metformin was administered intraperitoneally at a dose of $150 \mathrm{mg} / \mathrm{kg}$ body weight. Group IV, V and VI received Et-Ac, Pet-ether and chloroform partitionates of $O$. sanctum leaf extracts. The blood samples were analyzed for blood glucose content at $0,2,6,16$, and 24 hours, respectively.

For Oral glucose tolerance test (OGTT) animals were divided into five groups. Group VII to Group XI was prepared for testing hypoglycemic effects after glucose induced hyperglycemia in rats. Group VII received only vehicle (DMSO). Group VIII was selected for positive control, which did not receive either metformin, or plant extracts. Group IX stands for metformin control. Group X and XI received Pet-ether and Et-Ac partitionates of $O$. sanctum leaf extracts that had significant antihyperglycemic activity. After fasting for overnight, a baseline blood glucose level was estimated (0 minutes). Without delay, a glucose solution ( $2 \mathrm{gm} / \mathrm{kg}$ body weight) was administered by gavage. At the same time 
standard drug and plant extracts were administered intraperitoneally to the respective animal groups. Five more blood samples were taken at 30, 60, 90, 150, 270 minutes after glucose administration and blood glucose level was estimated in all the experiments by using glucometer (Bioland-423, Germany).

\subsection{Collection of serum and determination of serum total cholesterol (TC), serum triglycerides (TG), SGOT and SGPT}

After completing blood glucose level estimation rats were sacrificed and about 3-5 $\mathrm{ml}$ of blood was collected directly from heart by syringes, centrifuged at $4000 \mathrm{rpm}$ for 10 minutes and the serum was preserved to examine, TC, TG, SGOT and SGPT concentrations by UV spectrophotometric method (Shimadzu UV-1200, Tokyo, Japan), using wet reagent diagnostic kits according to manufacturer's protocol. At the same time liver tissue was also collected for the estimation of glycogen content in liver.

\subsection{Estimation of glycogen content in liver}

Glycogen content in liver was measured according to spectrophotometric determination of glycogen with o-toluidine reagent. It utilizes the o-toluidine glucose coupling reactions for the estimation of glycogen after trichloroacetic acid extraction, precipitation by alcohol and hydrolysis.

\subsection{Phytochemical screening methods}

Phytochemical tests have been performed according to the literature by Nayak and Pereira [15]:

(i) Test for saponins: $300 \mathrm{mg}$ of extract was boiled with $5 \mathrm{ml}$ water for two minutes. The mixture was cooled and mixed vigorously and left for three minutes. The formation of frothing indicated the presence of saponins.

(ii) Test for tannins: To an aliquot of the extract, sodium chloride is added to make to $2 \%$ strength. Then it is filtered and mixed with $1 \%$ gelatin solution. Precipitation indicated the presence of tannins.

(iii) Test for Triterpenes: $300 \mathrm{mg}$ of extract was mixed with $5 \mathrm{ml}$ chloroform and warmed for 30 minutes. The chloroform solution was then treated with a small volume of concentrated sulphuric acid and mixed properly. The appearance of red color indicated the presence of triterpenes.

(iv) Test for alkaloids: $300 \mathrm{mg}$ of extract was digested with $2 \mathrm{M} \mathrm{HCl}$. Acidic filtrate was mixed with amyl alcohol at room temperature, and examined the alcoholic layer for the pink colour which indicated the presence of alkaloids.

(v) Test for flavonoids: The presence of flavonoids was determined using $1 \%$ aluminium chloride solution in methanol, concentrated $\mathrm{HCl}$, magnesium turnins, and potassium hydroxide solution. 


\subsection{Statistical analysis}

Data were expressed as mean \pm standard error of mean (SEM). Statistical comparisons were performed by one-way analysis of variance (ANOVA), followed by Scheffe's posthoc test or students paired or unpaired t-test where appropriate. Results were considered to be significant when $p$ values were less than $0.05(\mathrm{p}<0.05)$. Statistical calculations and the graphs were prepared using GraphPad Prism version 4.00 for Windows (GraphPad Software, San Diego, CA, USA, www.graphpad.com).

\section{Results}

The effect of the different fractions of $O$. sanctum on the fasting blood glucose (FBG) level, serum total cholesterol (TC), serum triglyceride (TG), SGOT, SGPT levels and glycogen content in liver were investigated in the control and alloxan-induced diabetic rats using metformin $\mathrm{HCl}$ as standard antidiabetic agent.

\subsection{Effect of different fractions of $O$. sanctum on fasting blood glucose (FBG) level in diabetic rats}

The mean blood glucose concentration of control and different fractions of $O$. sanctumtreated animals were estimated on the 2, 6, 16, and 24 hours, respectively as shown in Fig 1. Their baseline glucose concentration was also measured. Et-Ac fraction of $O$. sanctum reduced blood glucose level to $74.67,48.95,19.81$ and $30.86 \%$ at 2,6,16 and 24 hours,

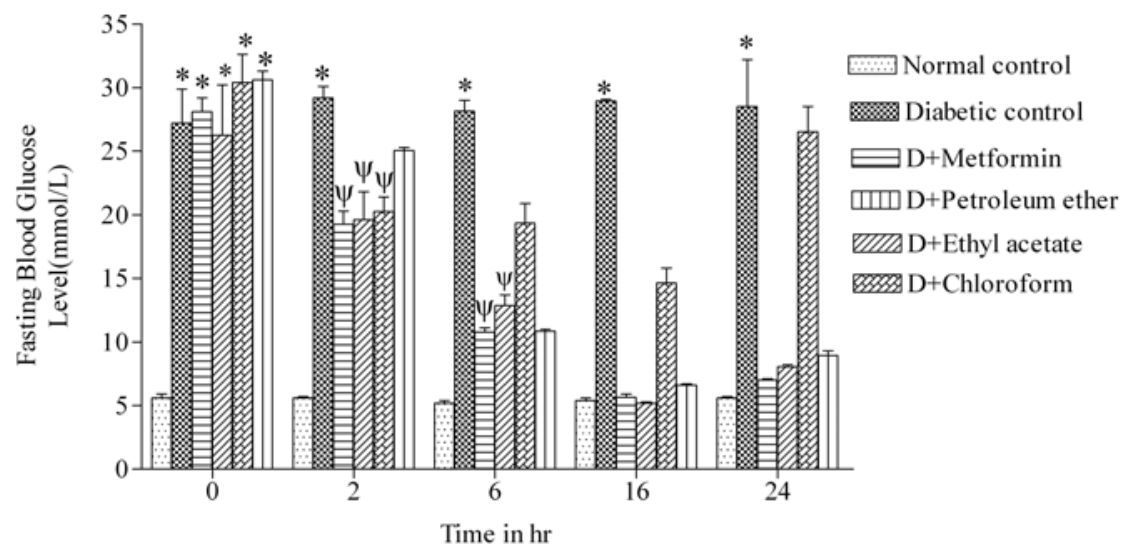

Fig. 1. Effect of different fractions of $O$. sanctum on the FBG level on diabetic rats compared to normal rats. * indicates significant change in blood glucose level compared with normal control group $(p<0.05) . \psi$ indicates significant changes in FBG level in diabetic rats after treatment $(p<0.05)$. The results are expressed as mean \pm SEM. 
respectively. Maximum reduction of blood glucose level of $80.19 \%$ was observed on 16 hour of the experiment. $\mathrm{CHCl}_{3}$ fraction of $\mathrm{O}$. sanctum showed reduction of blood glucose level to $66.18 \%, 63.24 \%, 47.88 \%$ and $87.34 \%$ at $2,6,16$ and 24 hours, respectively. Maximum reduction of blood glucose level of 52.12\% was also observed on 16 hour during the 24 hour experimental period. Pet-ether fraction of $O$. sanctum reduced blood glucose level to $81.86 \%, 35.45 \%, 21.56 \%$ and $29.24 \%$ at $2,6,16$ and 24 hours, respectively. Here Maximum reduction of blood glucose level of $78.44 \%$ was also observed on 16 hour of the experiment.

In case of alloxan induced diabetic rats metformin reduced blood glucose level to $68.50 \%, 38.43 \%, 20.10 \%$, and $24.91 \%$ at $2,6,16$ and 24 hours, respectively. So metformin caused maximum reduction of blood glucose level of $79.90 \%$ on 16 hour of the experiment.

\subsection{Effect of different fractions of $O$. sanctum on TC and TG level in diabetic rats}

The mean serum total cholesterol and triglyceride level of control and treated animals after 24 hours are shown in Fig. 2 and Fig. 3 respectively. Hypolipidemia was observed in animals treated with the different fractions. In case of the effects of standard metformin $\mathrm{HCl}$ and different fractions of $O$. sanctum on total cholesterol level in diabetic rats, the metformin, Et-Ac, Pet-ether and $\mathrm{CHCl}_{3}$ fractions of $O$. sanctum reduced total cholesterol level to $46.55 \%, 45.51 \%, 67.32 \%$ and $76.22 \%$, respectively. Maximum reduction of serum total cholesterol level of $54.49 \%$ was found for Et-Ac fraction of O. sanctum. During the effects of metformin and different fractions of $O$. sanctum on serum triglyceride level in diabetic rats, the metformin, Et-Ac, Pet-ether and $\mathrm{CHCl}_{3}$ fractions of O. sanctum reduced serum triglyceride level to $38.98 \%, 20.22 \%, 26.50 \%$ and $42.16 \%$, respectively. Maximum reduction of serum triglyceride level of $79.78 \%$ was found for EtAc fraction of $O$. sanctum.

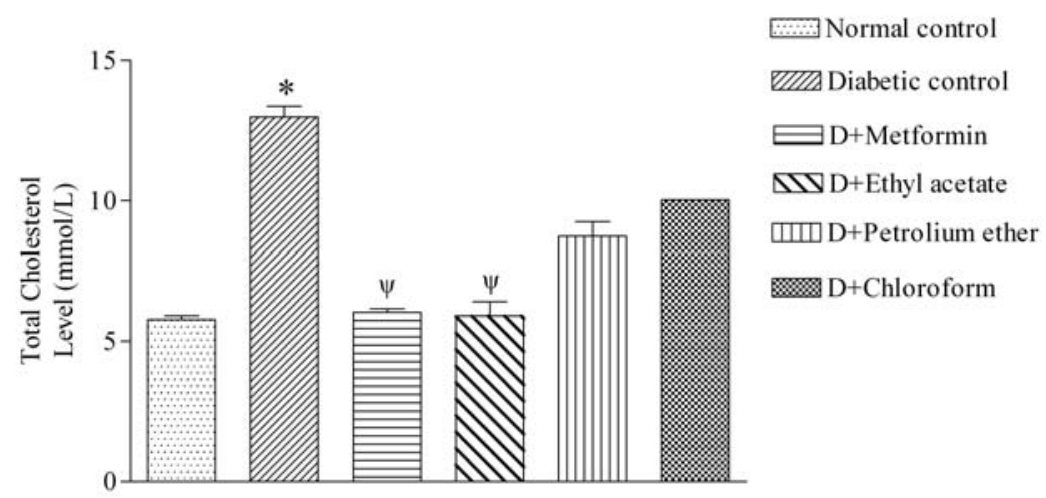

Fig. 2. Effect of different fractions of $O$. sanctum on the total cholesterol level in diabetic rats. * indicates significant change compared with normal control group $(\mathrm{p}<0.05)$. $\psi$ indicates significant changes in diabetic rats after treatment $(\mathrm{p}<0.05)$. The results are expressed as mean \pm SEM. 


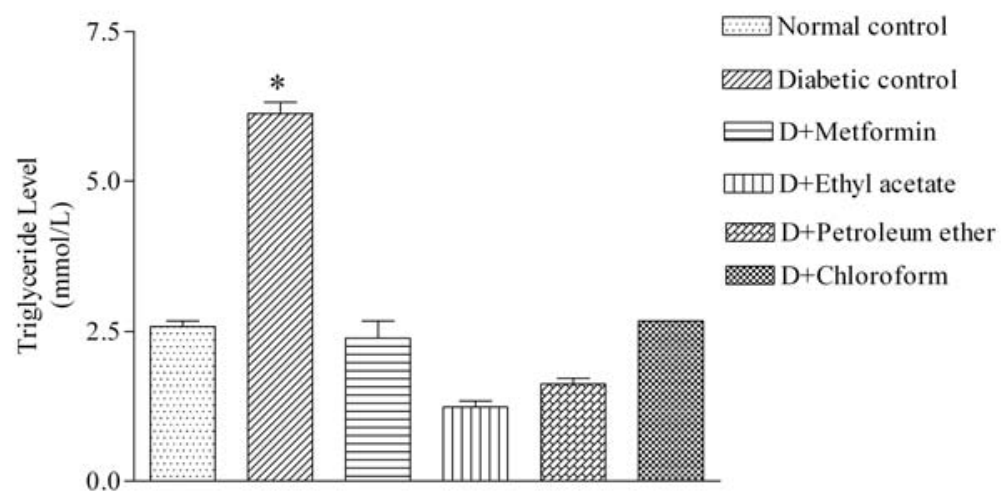

Fig. 3. Effect of different fractions of $O$. sanctum on the triglyceride level in diabetic rats. * indicates significant change compared with normal control group $(p<0.05)$. The results are expressed as mean $\pm \mathrm{SEM}$.

\subsection{Effect of experimental plant fractions on the level of glycogen in diabetic rats}

In this study it is found that the level of glycogen in liver is reduced to $49.34 \%$ in diabetic rats as compared to the normal control group. Treatment of diabetic rats with metformin standard, Et-Ac and Pet-ether fractions of $O$. sanctum the level of glycogen content was improved to $86.31 \%, 90.84 \%$ and $99.58 \%$, respectively as shown in the Fig. 4 .

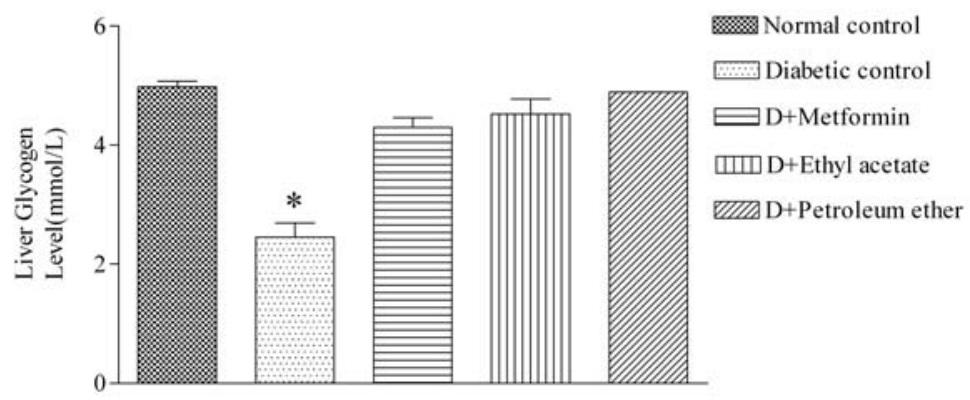

Fig. 4. Effect of different fractions of $O$. sanctum on the glycogen level in liver on diabetic rats compared to normal rats. * indicates significant difference $(p<0.05)$ from normal control. The results are expressed as mean $\pm \mathrm{SEM}$.

\subsection{Effect of different fractions of O. sanctum on liver enzymes (SGOT, SGPT) in diabetic rats}

In diabetic rats SGOT and SGPT levels were raised to $60.86 \%$ and $19.85 \%$ respectively in comparison to normal rats. Following intraperitoneally administration of different plant fractions, SGOT and SGPT levels were significantly reduced as shown in the Table 1. 
Table 1. Effect of different fractions of $O$. sanctum ethanolic extract on SGPT and SGOT level. * indicates significant difference $(p<0.05)$ from normal control group. $\psi$ indicates significant difference $(p<0.05)$ from the diabetic control. Data are expressed as mean \pm SEM.

\begin{tabular}{lcc}
\hline \multicolumn{1}{c}{ Group } & $\begin{array}{c}\text { SGPT } \\
(\text { Unit/ml) }\end{array}$ & $\begin{array}{c}\text { SGOT } \\
(\text { Unit } / \mathrm{ml})\end{array}$ \\
\hline Normal Control & 21.4 & 18.0 \\
Diabetic Control & 26.7 & $46.0^{*}$ \\
D+Metformin & $11.5 \psi$ & $25.2 \psi$ \\
D+Ethyl acetate & 17.6 & $11.4 \psi$ \\
D+Petroleum ether & $14.3 \psi$ & $10.3 \psi$ \\
\hline
\end{tabular}

\subsection{Effect of different fractions of $O$. sanctum on fasting blood glucose (FBG) level in the glucose-induced hyperglycemic rats}

Et-Ac fraction of $O$. sanctum showed reduction of blood glucose level to $89.09 \%, 91.53 \%$, $66.89 \%$, and $80.55 \%$ and Pet -ether fraction of $O$. sanctum reduced blood glucose level to $78.18 \%, 75.13 \%$, and $72.97 \%$ at $30 \mathrm{~min}, 60 \mathrm{~min}$, and $90 \mathrm{~min}$, respectively in glucose induced hyperglycemic rats as shown in the Fig. 5. So it was found that both Et-Ac and Pet-ether fraction showed maximum reduction of $33.11 \%$ and $27.03 \%$, respectively at 90 min of the experimental period.

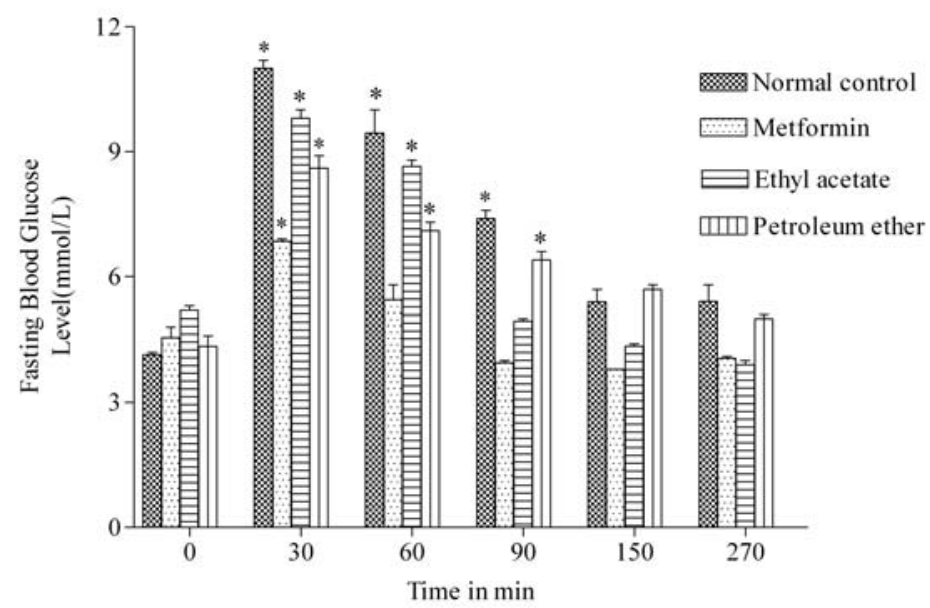

Fig. 5. Effect of different fractions of $O$. sanctum on the glucose-induced hyperglycemia in normal rats. * indicates significant changes in FBG level compared to normal rats after treatment $(p<0.05)$. The results are expressed as mean $\pm \mathrm{SEM}$.

\subsection{Phytochemical screening test result}

The phytochemical screening test result is shown in the Table 2. 
Table 2. The phytochemical constituents of the experimental plant fractions obtained by phytochemical screening tests.

\begin{tabular}{lccccc}
\hline Partitionates & Saponin & Tannins & Triterpines & Alkaloids & Flavanoids \\
\hline Chloroform & - & + & - & - & + \\
Ethyl acetate & - & + & + & - & + \\
Petroleum ether & + & + & + & - & + \\
\hline
\end{tabular}

(-) -not detected; (+)-detected.

\section{Discussion}

Diabetes mellitus is possibly the world's largest growing metabolic disease, and as the knowledge on the heterogeneity of this disorder is advanced, the need for more appropriate therapy increases [16]. Traditional plant medicines are used throughout the world for a range of diabetic presentations. The study of such medicines might offer a natural key to unlock a diabetologist's pharmacy for the future.

In the light of the literature on $O$. sanctum, we made an attempt for the first time to study the effect of $O$. sanctum ethanolic extract partitionates in normoglycemic and hyperglycemic rats.

The significant anti diabetic activity of Et- Ac, Pet-ether and chloroform fractions of $O$. sanctum as shown in Fig. 1 may be due to the presence of hypoglycemic saponins, triterpines, and flavonoids. It could be conceived that the plant extracts may also contain some biomolecules that may sensitize the insulin receptor to insulin or stimulates the $\beta$ cells of islets of langerhans to release insulin which may finally lead to improvement of carbohydrate metabolizing enzymes towards the re-establishment of normal blood glucose level.

Hypercholesterolemia and hypertriglyceridemia have been reported to occur in diabetic rats [17-19]. Intraperitoneal administration of partitionates of ethanol extract of leaves of $O$. sanctum resulted in a significant reduction of serum lipid levels in rats with hyperlipidemia viz. triglyceride and total cholesterol (Fig. 2 and 3). Flavonoids are known for their diverse biological activities including hypolipidemic activity resulting from their antioxidant activity [20]. O. sanctum partitionates showed the presence of flavonoids and related phenolic compounds (Table 2). Such dual property has also been reported in methanol extract of Prunus dadidiana (Rosaceae) and its flavonoid constituent, prunin [21]. Induction of diabetes with alloxan was associated with decrease in hepatic glycogen, which could be attributed to the decrease in the availability of the active form of enzyme glycogen synthetase probably because of low levels of insulin [22, 23]. In the present study, $O$. sanctum restored the depressed hepatic glycogen levels possibly by increasing the level of insulin. Our results showed that supplementation of diabetic rats with plant fractions resulted in significant elevation in hepatic glycogen content. Decreased activities of the enzymes involved in glucose homeostasis in liver and kidney such as hexokinase has been reported in diabetic animals resulting in depletion of liver and muscle glycogen 
content [24]. Treatment with plant extracts might increase the level of enzyme to the control level indicating an over-all increase in glucose influx. The exact mechanism of action needs further investigation.

There was a significant rise in serum GOT and GPT levels in diabetic rats, which could relate to excessive accumulation of amino acids (glutamate and alanine) in the serum of diabetic animals as a result of amino acids mobilization from protein stores [25]. The higher levels of GOT and GPT, may give rise to a high concentration of glucose. In other words, the gluconeogenic action of GOT and GPT plays the role of providing new supplies of glucose from other sources such as amino acids. Following intraperitoneally administration of different plant fractions, SGOT and SGPT levels were significantly reduced (Table 2).

Oral glucose tolerance test (OGTT) measures the body's ability to use glucose, the body's main source of energy [26]. It can be used to diagnose prediabetes and diabetes. In our study, it is found that various fractions have also hypoglycemic effect in glucose induced hyperglycemic rats. This may be due to the presence of hypoglycemic flavonoids, triterpines or saponin glycosides that also requiring further investigation.

\section{Conclusion}

This study is unique in that plant fractions cause rapid induction of hypoglycemia and hypolipidemia as well as possesses hepatoprotective effect in diabetic rats. In the light of our pharmacological studies $O$. sanctum leaf extracts can be useful, at least as an adjunct, in the therapy of diabetes, a condition in which hyperglycemia and hyperlipidemia coexist quite often. We need further study to determine the mechanism of action and to isolate the active principles responsible for antidiabetic activity.

\section{Acknowledgements}

The present work was supported by the National Science and Information and Communication Technology (NSICT), Dhaka, Bangladesh for financial assistance to the first author and the authors would like to extend their gratitude to the Director, Animal Research Centre (ARC), ICDDR, B for providing necessary facilities.

\section{References}

1. Diabetes action now (WHO, Geneva, IDF-Brussels, 2004) pp. 1-20.

2. R. Sicree, J. Shaw, and P. Zimmet, Diabetes and impaired glucose tolerance. In: Gan D, ed. Diabetes Atlas. 3rd ed. (Belgium: International Diabetes Federation, 2006) pp. 15-103.

3. G. Roglic, N. Unwin, P. H. Bennett, C. Mathers, J. Tuomilehto, S. Nag, V. Connolly, and H. King, Diabetes Care 28, 2130 (2005). doi:10.2337/diacare.28.9.2130

4. V. Mohan, C. S. Shanthirani, M. Deepa, R. Deepa, R. I. Unnikrishnan, and M. Datta, J Assoc Physicians India 54, 113 (2006).

5. C. L. Morgan, C. J. Currie, and J. R. Peters, Diabetes Care 23, 1103 (2000). doi:10.2337/diacare.23.8.1103

6. K. Gu, C. C. Cowie, and M. I. Harris, Diabetes Care 21, 1138 (1998). doi:10.2337/diacare.21.7.1138 
7. G. Roglic, Diabetes mortality. In: Gan D, ed. Diabetes atlas. 3rd ed. (Belgium: International Diabetes Federation, 2006) pp. 219-36.

8. C. J. Bailey and C. Day, Diabetes Care 12, 553 (1989). doi:10.2337/diacare.12.8.553

9. J. K. Grover, S. Yadav, and V. Vats, J. Ethnopharmacol. 81, 81(2002). doi:10.1016/S0378-8741(02)00059-4

10. N. Singh, R. Nath, and M. L Gupta, Q. J. Crude Drug Res. 18, 86 (1980).

11. K. Kirtikar and B. Basu, In Indian Medicinal Plants, (Periodical Expert Book Agency, New Delhi, India: 1993) pp. 3-6.

12. H. Wagner and H. Winterhoff, Phytomedicine 1, 63 (1994).

13. P. K. Warier. In Indian Medicinal Plants, (Eds PK Warrier, VPK Nambiar \& C Ramankutty. Madras, India: Orient Longman.1995a) pp. 48-51.

14. K. P. Bhargava and N Singh, Ind. J. of Med. Res. 73, 443 (1981).

15. B. S. Nayak and L. M. Pinto Pereira, BMC Complement Altern. Med. 6, 41 (2006). doi:10.1186/1472-6882-6-41

16. C. J. Baily and P. R. Flatt, Ind. Biotechnology 6, 139 (1986).

17. A. Riyad, S. Abdul-Ghani Abdul-Salam, and S. M. Suleiman, Planta. Med. 54, 286 (1988). doi:10.1055/s-2006-962434

18. S. P. Tarfa, P. K. Joseph, and K.T. Augusti, Curr Sci. 57, 32 (1988).

19. S. R. Sharma, S. K. Dwivewdi, and D. Swarup, Ind. J. of Exp Biol. 34, 372 (1996).

20. I. B. Afanas'ev, E. A. Ostrachovitch, N. E. Abramova, and L. G. Korkina, Biochem Pharmacol. 80, 627 (1995). doi:10.1016/0006-2952(95)00173-W

21. J. S. Choi, and O. H. Yokozawa, J. Nat. Prod. 54, 218 (1991). doi:10.1021/np50073a022

22. A. H. Gold, J. Biol. Chem. 245, 903 (1970).

23. R. K. Goel, M. P. Mahajan, and S. K. Kulkarni, J. Pharm. Pharm. Sci. 7, 80 (2004).

24. J. K. Grover, V. Vats, and S. S. Rathi, J. Ethnopharmacol. 73 (3), 461 (2000). doi:10.1016/S0378-8741(00)00319-6

25. V. Colev, M. Badescu, and I. Paduraru, Rom J. Inter Med. 32, 71 (1994).

26. Du Vigneaud, and W. G. Karr, J. Biol. Chem. 66, 281(1925). 